\title{
A field experiment comparing mechanical demands of two pruners for flower cutting
}

\author{
Berrio $\mathrm{S}^{\mathrm{a}}$, Barrero $\mathrm{LH}^{\mathrm{a}}$, and Quintana $\mathrm{LA}^{\mathrm{a}}$ \\ ${ }^{a}$ Department of Industrial Engineering, Pontificia Universidad Javeriana, Carrera 7 \# 40-62 Bogotá, Colombia
}

\begin{abstract}
Manual flower cutting requires repetitive forceful movements that may explain the high incidence of upperextremity MSDs among workers of the Colombian flower industry. Force demands may be caused by the use of pruners that have not been designed for Colombian workers. We conducted a repeated measures field experimental study $(n=16,69 \%$ women, 20 to 60 years old) to compare the mechanical demands during rose cutting of two different pruners (Traditional vs. alternative pruner) at two different cutting heights (elbow vs. over-shoulder). Demands included the perceived comfort, perceived effort and upper limb's muscular activity and posture. Workers perceived their traditional pruner as more comfortable but requiring more effort than the prototype tool. No benefits were observed regarding ulnar deviation with the alternative pruner. The wrist extension and related muscular activity of the wrist extensors were higher with the prototype pruner. The prototype pruner resulted in reduced muscular load of the wrist flexors; the biceps when working at the elbow height; and the deltoids when working at the shoulder height. However, Moderate results may be explained by the lack of time for workers' adaptation to the alternative tool. New design developments must focus on reducing extension and ulnar deviation of the wrist.
\end{abstract}

Keywords: Upper extremity, electromyography, electrogoniometer, hand tool, mechanical exposures.

\section{Introduction}

Colombia is the second largest flower exporter of the world [1]. The floriculture industry employs over 90000 workers in production areas [2]. However, this industry also has one of the highest prevalence of recorded occupational musculoskeletal disease in Colombia, mainly those that affect the upper limbs [3].

Manual flower cutting is one of the tasks that are said to impose higher mechanical demands to workers because it requires repetitive forceful movements [4]. Force demands may be caused in part or exacerbated by the common use of tools that are not made for the Colombian working population. These traditional tools require workers to initiate forceful movements with almost fully extended fingers and require frequent excursions in the ulnar direction which may impose large mechanical loads to workers [5]. In this study, we tested an alternative design pruner that is expected to provide better mechanical advantage to the worker; that would result in reduced muscular load and more neutral postures of the upper-extremity.

\section{Methods}

\subsection{Design}

A repeated measures experimental study was conducted in the field to test the differences in mechanical demands of two rose pruners, the traditional Felco-2 $\AA$ and an alternative prototype designed by our group [6] (Figure 1). The Felco-2 is a Swiss pruner that is most frequently used among Colombian workers. Sixteen workers (11 women, age from 20 to 60 years old) were assessed while executing their regular cutting tasks at fixed cutting frequencies, and at two cutting heights (elbow and above-shoulder height) according to real working conditions. Both tools used brand-new blades. Workers were not allowed to test the prototype prior to the experiments as the design was thought to require no training or previous experience with the tool. All participants

\footnotetext{
Corresponding author. E-mail: lopehugo@javeriana.edu.co
} 
signed an informed consent; and all protocols were approved by the ethics committee of Pontificia Universidad Javeriana, Bogota, Colombia.

\subsection{Dependent variables}

Workers' self-reported comfort and experience with the tool were documented immediately after each experimental treatment. Also, posture of the wrist and elbow were recorded using an electrogoniometer (Biometrics, UK). Lastly, muscular activity was assessed using surface EMG (Biometrics, UK) following standardized guidelines [7,8]. Assessed muscles included the extensor carpi ulnaris (ECU), extensor carpi radialis (ECR), flexor carpi ulnaris (FCU), flexor carpi radialis (FCR), biceps and deltoids of the dominant arm.

\subsection{Data analysis}

Posture signals were processed analyzed using the DataLINK software (Biometrics, UK). Mean and percentiles 10, 50 and 90 were estimated for each worker and experimental treatment in preparation for statistical analysis. Similarly, muscular activity signals were processed and digitally analyzed using MATLAB. The Amplitude Probability Distribution Function was estimated for the RMS (200 ms moving window) normalized EMG signal for each worker and experimental treatment. Statistical analyses were executed in SPSS 11.0. Interaction and main effects of pruner and working height on the exposure variables of interest were tested using univariate lineal repeated measure analyses with a compound symmetry co-variance matrix.

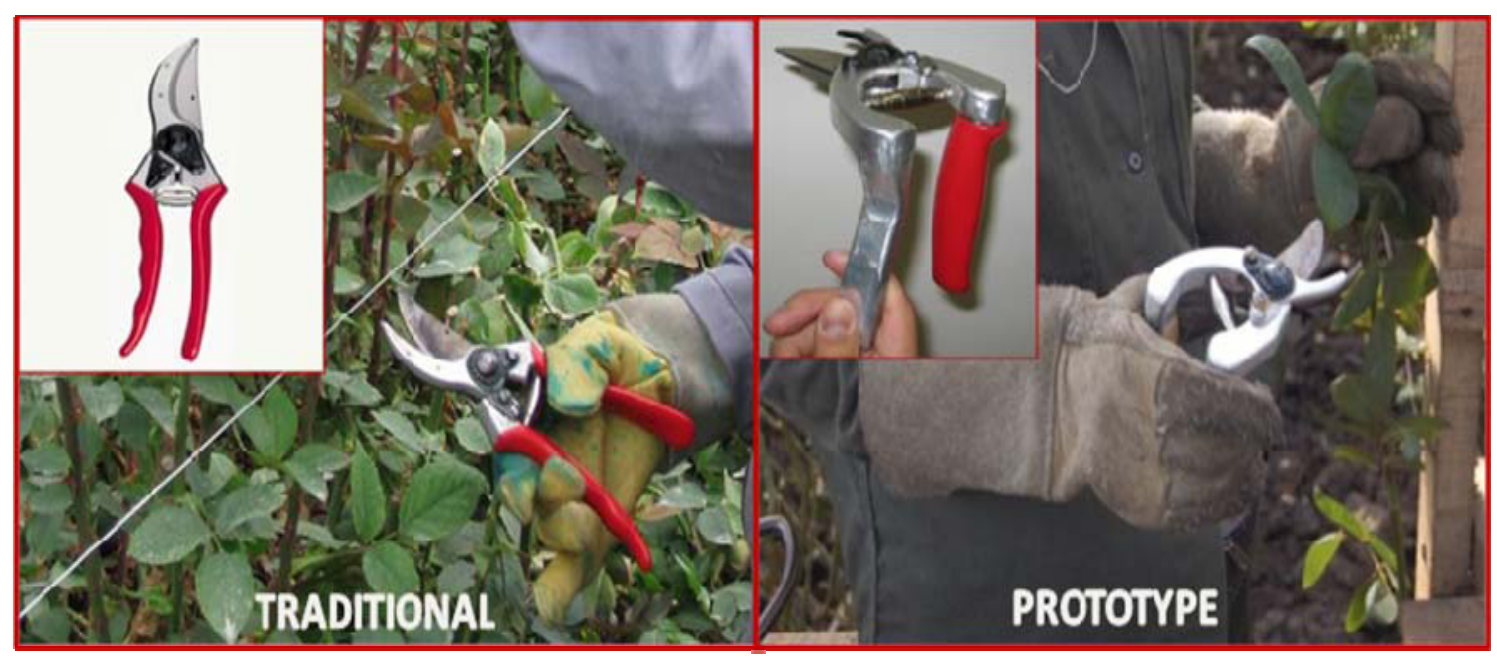

Figure 1. Flower cutting hand tools being compared in the present study

\section{Results}

Self-reports indicate that workers perceived their traditional pruner as more comfortable than the prototype tool $(p<0.01)$; however, they also reported less effort using the prototype pruner $(\mathrm{p}<0.01)$. Workers reported having difficulties with the mechanism to secure the alternative pruner and could not adapt to the moving handle.

Most assessed postures resulted similar for both tools. However, the extension of the wrist for both working heights were larger when using the proto- type pruner $(\mathrm{p}<0.01)$. This is consistent with the observed muscular activity, which was also larger for the wrist extensors, especially when using the prototype pruner at the elbow height. The prototype pruner appears to result in reduced muscular load of the wrist flexors; the biceps when working at the elbow height; and the deltoids when working at the shoulder height. Surprisingly, we did not observe major benefits in the degree of ulnar deviation with the prototype pruner in spite of the design efforts to achieve this effect (Figures 2 and 3 ). 


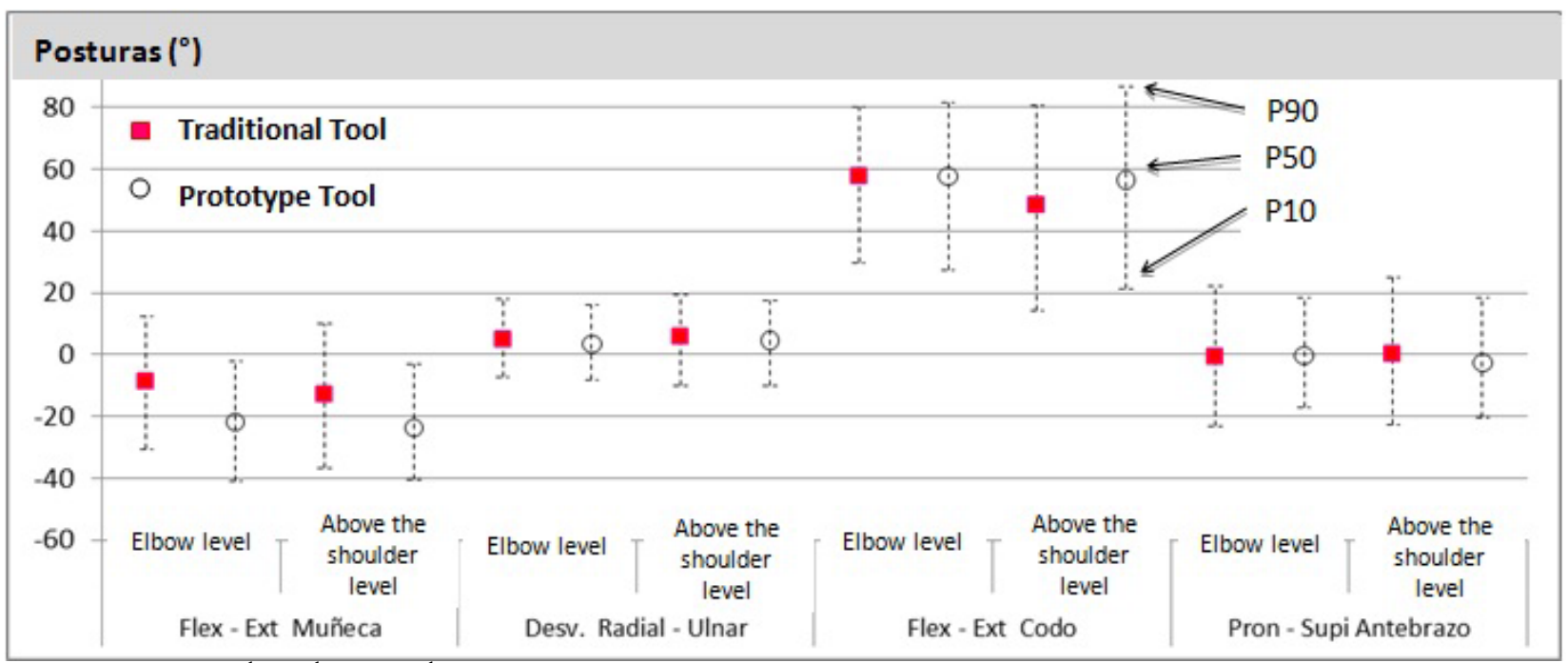

Figure $2.10^{\text {th }}, 50^{\text {th }}$ and $90^{\text {th }}$ percentiles of posture associated to two pruners at two working height

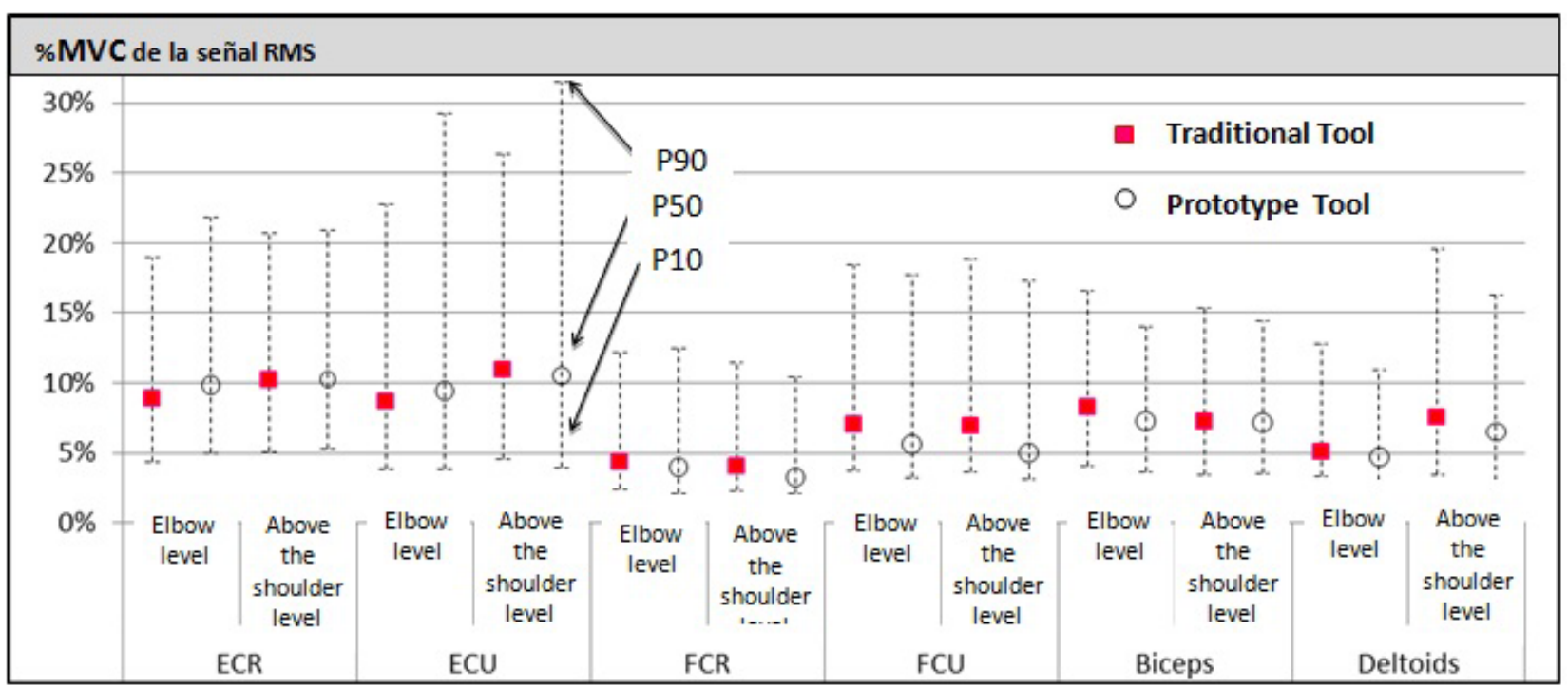

Figure $3.10^{\text {th }}, 50^{\text {th }}$ and $90^{\text {th }}$ percentiles of muscle activity associated to two pruners at two working heights

\section{Discussion}

There were only moderate differences in mechanical demands between the two tested pruners. Expected benefits from the prototype pruner may have been hidden by the lack of time for workers' adaptation to the new alternative tool and miscellaneous characteristics of the tool not related to shape and dimension that are still to be improved prior to the final model. Results do not allow recommending the use of the prototype yet. New design developments must focus on reducing extension of the wrist and better capitalizing potential benefits regarding ulnar deviation. 


\section{References}

[1] Tenjo, F., Montes, E. \& Martínez, J. (2006). Comportamiento reciente (2000-2005) del sector floricultor colombiano. In Presentaciones y discursos, codirectores, Banco de la República [In Spanish], Bogotá, Colombia: Colombian Central Bank.

[2] Asocolflores. (2008). Floricultura Colombiana Estadísticas 2007, in Datos de la Floricultura.

[3] Ministerio de la Protección Social (MPS). (2007). Informe de enfermedad profesional en Colombia 2003-2005 [In Spanish], Bogotá, Colombia: Imprenta Nacional de Colombia.

[4] Barrero, L.H., Rodríguez, L.E., Suárez, N., Camacho A.M., Ceballos, C., Quintana, L.A., et al. (2010). Upperextremity muscle activity during flowers cutting and packing in a Colombian working population. PREMUS 2010. Angers, France.

[5] Blackwell, J.R., Kornatz, K.W., \& Heath, E.M. (1999). Effect of grip span on maximal grip force and fatigue of flexor digitorum superficialis. Applied Ergonomics, 30, 401-405.

[6] Córdoba, J. \& Fajardo, J. (2007). Undergraduate thesis in Industrial Design: Herramienta para Corte de Rosas [In Spanish].

[7] Hermens JH, Freriks B, et al. (1999). European Recommendations for Surface Electromyography. Roessingh Research and Development.

[8] Perotto AO, et al. (2005). Anatomical guide for the electromyographer: The limbs and trunk. Springfield, Illinois: Charles $\mathrm{C}$ Thomas. 